\title{
A CASE OF COMBINED RADIAL AND AXIAL HEAT FLOW IN COMPOSITE CYLINDERS*
}

\author{
BY \\ V. R. THIRUVENKATACHAR \\ Central College, Bangalore, India** \\ AND \\ B. S. RAMAKRISHNA \\ Indian Institute of Science, Bangalore, India
}

Introduction. Although several problems of heat flow in composite cylinders have been studied, all the cases considered treat the heat flow in the radial direction only $[1,2,3]$. The case of combined radial and axial heat flow in composite cylinders presents an interesting boundary value problem which has also considerable significance in the theory of vibrations and propagation of electromagnetic waves $[4,5,6]$. In this paper, we consider a case of combined radial and axial heat flow in the unsteady state in finite cylinders composed of two coaxial parts of different materials. The temperature distribution in the cylinder at any instant under the assumed boundary and initial conditions has been obtained by the use of the Laplace transformation. The procedure is illustrated by a numerical calculation in a particular case.

The Problem. Composite cylinder made of two different materials, the inner cylinder $0 \leq r \leq a$ and the outer cylinder $a \leq r \leq b$ having thermal conductivity and diffusivity coefficients $\epsilon_{1}$ and $k_{1}$ and $\epsilon_{2}$ and $k_{2}$ respectively. $\dagger$ Boundary conditions: The flat ends of the cylinder $x=0$ and $x=l \mathrm{kept}$ at zero temperature with the outer surface insulated and perfect thermal contact at $r=a$ between the two coaxial parts. Initially the cylinder is assumed to be heated to constant unit temperature. Required the temperature distribution in the cylinder for any time $t>0$ (see Fig. 1).

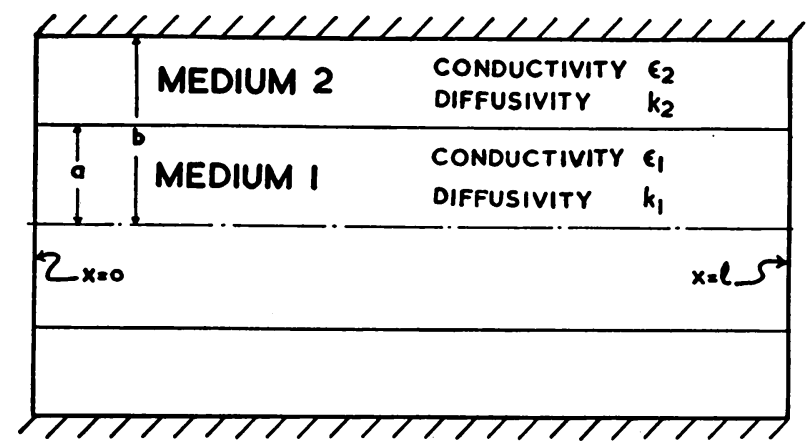

FIG. 1. Composite cylinder, the inner cylinder having conductivity and diffusivity coefficients $\epsilon_{1}$ and $k^{1}$ respectively and the outer $\epsilon_{2}$ and $k_{2}$.

*Received December 1, 1951.

**Now at Defense Science Organisation, Ministry of Defense, New Delhi, India.

†Throughout this article the subscripts 1 and 2 refer to the regions $0 \leq r \leq a$ and $a \leq r \leq b$ respectively. 
Method of solution. The boundary value problem for the temperatures $u_{1}$ and $u_{2}$ in the inner and outer cylinders may be stated as follows:

$$
\begin{array}{ll}
\frac{\partial u_{1}}{\partial t}=k_{1}\left(\frac{\partial^{2} u_{1}}{\partial r^{2}}+\frac{1}{r} \frac{\partial u_{1}}{\partial r}+\frac{\partial^{2} u_{1}}{\partial x^{2}}\right), & 0 \leq r \leq a, \\
\frac{\partial u_{2}}{\partial t}=k_{2}\left(\frac{\partial^{2} u_{2}}{\partial r^{2}}+\frac{1}{r} \frac{\partial u_{2}}{\partial r}+\frac{\partial^{2} u_{2}}{\partial x^{2}}\right), & a \leq r \leq b,
\end{array}
$$

with the initial conditions

$$
u_{1}(r, x, 0)=u_{2}(r, x, 0)=1,
$$

and the boundary conditions

$$
\begin{aligned}
u_{1}(r, 0, t) & =u_{2}(r, 0, t)=0, \\
u_{1}(r, l, t) & =u_{2}(r, l, t)=0, \\
u_{1}(a, x, t) & =u_{2}(a, x, t), \\
\epsilon_{1} \partial u_{1}(a, x, t) / \partial r & =\epsilon_{2} \partial u_{2}(a, x, t) / \partial r, \\
\epsilon_{2} \partial u_{2}(b, x, t) / \partial r & =0 .
\end{aligned}
$$

We require the temperature functions $u_{1}(r, x, t)$ and $u_{2}(r, x, t)$ satisfying the equations from (1) to (8) inclusive.

Let

$$
\begin{aligned}
& U_{1}(r, x, s)=\int_{0}^{\infty} u_{1}(r, x, t) e^{-s t} d t \\
& U_{2}(r, x, s)=\int_{0}^{\infty} u_{2}(r, x, t) e^{-s t} d t
\end{aligned}
$$

be the Laplace transforms of $u_{1}$ and $u_{2}$. The transforms $U_{1}(r, x, s)$ and $U_{2}(r, x, s)$ will then satisfy the equations

$$
\begin{aligned}
& \frac{\partial^{2} U_{1}}{\partial r^{2}}+\frac{1}{r} \frac{\partial U_{1}}{\partial r}+\frac{\partial^{2} U_{1}}{\partial x^{2}}-\frac{s}{k_{1}} U_{1}=-\frac{1}{k_{1}}, \\
& \frac{\partial^{2} U_{2}}{\partial r^{2}}+\frac{1}{r} \frac{\partial U_{2}}{\partial r}+\frac{\partial^{2} U_{2}}{\partial x^{2}}-\frac{s}{k_{2}} U_{2}=-\frac{1}{k_{2}},
\end{aligned}
$$

and the boundary conditions

$$
\begin{aligned}
U_{1}(r, 0, s) & =U_{2}(r, 0, s)=0, \\
U_{1}(r, l, s) & =U_{2}(r, l, s)=0, \\
U_{1}(a, x, s) & =U_{2}(a, x, s), \\
\epsilon_{1} \partial U_{1}(a, x, s) / \partial r & =\epsilon_{2} \partial U_{2}(a, x, s) / \partial r \\
\partial U_{2}(b, x, s) / \partial r & =0 .
\end{aligned}
$$


In order that $U_{1}(r, x, s)$ and $U_{2}(r, x, s)$ may vanish at $x=0$ and $x=l$ as required by the boundary conditions (11) and (12), we expand $U_{1}$ and $U_{2}$ as well as the constants $-1 / k_{1}$ and $-1 / k_{2}$ on the right hand side of equations (9) and (10) in Fourier sine series

$$
\begin{aligned}
U_{1}(r, x, s) & =\sum_{n} V_{1 n}(r, s) \sin (n \pi x / l), \quad n=1,3,5, \cdots, \\
U_{2}(r, x, s) & =\sum_{n} V_{2 n}(r, s) \sin (n \pi x / l), \quad n=1,3,5, \cdots, \\
-\frac{1}{k_{1}} & =\sum_{n} b_{1 n} \sin (n \pi x / l), \quad n=1,3,5, \cdots, \\
-\frac{1}{k_{2}} & =\sum_{n} b_{2 n} \sin (n \pi x / l), \quad n=1,3,5, \cdots .
\end{aligned}
$$

For the sake of brevity, let

$$
\begin{aligned}
& \alpha_{n}^{2}=s / k_{1}+n^{2} \pi^{2} / l^{2} \\
& \beta_{n}^{2}=s / k_{2}+n^{2} \pi^{2} / l^{2}
\end{aligned}
$$

The radial functions $V_{1 n}$ and $V_{2 n}$ now satisfy respectively the equations

$$
\begin{aligned}
& \frac{\partial^{2} V_{1 n}}{\partial r^{2}}+\frac{1}{r} \frac{\partial V_{1 n}}{\partial r}-\alpha_{n}^{2}\left(V_{1 n}+\frac{b_{1 n}}{\alpha_{n}^{2}}\right)=0, \\
& \frac{\partial^{2} V_{2 n}}{\partial r^{2}}+\frac{1}{r} \frac{\partial V_{2 n}}{\partial r}-\beta_{n}^{2}\left(V_{2 n}+\frac{b_{2 n}}{\beta_{n}^{2}}\right)=0 .
\end{aligned}
$$

These have the solutions

$$
\begin{aligned}
& V_{1 n}(r, s)=A_{n} I_{0}\left(\alpha_{n} r\right)-\frac{b_{1 n}}{\alpha_{n}^{2}}, \\
& V_{2 n}(r, s)=B_{n} I_{0}\left(\beta_{n} r\right)+C_{n} K_{0}\left(\beta_{n} r\right)-\frac{b_{2 n}}{\beta_{n}^{2}},
\end{aligned}
$$

where $I_{0}$ and $K_{0}$ are respectively the modified Bessel functions of the first and the second kinds of zeroth order. The constants $A_{n}, B_{n}$, etc. are now to be determined from the boundary conditions (13), (14) and (15). Thus we obtain

$$
\begin{aligned}
& U_{1}(r, x, s)=\sum_{n}\left[\epsilon_{2} \beta_{n}\left(\frac{b_{1 n}}{\alpha_{n}^{2}}-\frac{b_{2 n}}{\beta_{n}^{2}}\right) \frac{\left\{I_{1}\left(\beta_{n} a\right) K_{1}\left(\beta_{n} b\right)-I_{1}\left(\beta_{n} b\right) K_{1}\left(\beta_{n} a\right)\right\} I_{0}\left(\alpha_{n} r\right)}{\Delta_{n}(s)}\right. \\
& U_{2}(r, x, s)=\sum_{n}\left[\epsilon_{1} \alpha_{n}\left(\frac{b_{1 n}}{\alpha_{n}^{2}}-\frac{b_{1 n}}{\alpha_{n}^{2}}\right] \sin \frac{n \pi x}{l},\right. \\
& \left.\beta_{n}^{2}\right) \frac{\left\{I_{0}\left(\beta_{n} r\right) K_{1}\left(\beta_{n} b\right)-I_{1}\left(\beta_{n} b\right) K_{0}\left(\beta_{n} r\right)\right\} I_{1}\left(\alpha_{n} a\right)}{\cdot} \\
& \left.-\frac{b_{2 n}}{\beta_{n}^{2}}\right] \sin \frac{n \pi x}{l},
\end{aligned}
$$


where

$$
\begin{aligned}
\Delta_{n}(s)=\epsilon_{2} \beta_{n} I_{0}\left(\alpha_{n} a\right)\left[I_{1}\left(\beta_{n} a\right) K_{1}\left(\beta_{n} b\right)\right. & \left.-I_{1}\left(\beta_{n} b\right) K_{1}\left(\beta_{n} a\right)\right] \\
& -\epsilon_{1} \alpha_{n} I_{1}\left(\alpha_{n} a\right)\left[I_{0}\left(\beta_{n} a\right) K_{1}\left(\beta_{n} b\right)+I_{1}\left(\beta_{n} b\right) K_{0}\left(\beta_{n} a\right)\right] .
\end{aligned}
$$

The temperature distribution functions $u_{1}(r, x, t)$ and $u_{2}(r, x, t)$ may be now obtained from $U_{1}(r, x, s)$ and $U_{2}(r, x, s)$ by the inversion integrals [7]

$$
\begin{aligned}
& u_{1}(r, x, t)=\frac{1}{2 \pi i} \int_{\gamma-i \infty}^{\gamma+i \infty} U_{1}(r, x, s) e^{s t} d s \\
& u_{2}(r, x, t)=\frac{1}{2 \pi i} \int_{\gamma-i \infty}^{\gamma+i \infty} U_{2}(r, x, s) e^{s t} d s
\end{aligned}
$$

The integrals in (21) and (22) may be expressed as $2 \pi i$ times the sum of the residues of the corresponding integrands at their poles. In evaluating the residue of $U_{1}(r, x, s)$ $\exp (s t)$ it will be noted that the first term in $U_{1}(r, x, s)$ has got the factor $\alpha_{n}^{2} \beta_{n} \Delta_{n}(s)$ in the denominator. It will be seen further that $\beta_{n}=0$ does not give rise to a pole since the expression remains finite (on account of the singularity of $K_{1}$ at the origin) as $\beta_{n} \rightarrow 0$ so that the only poles are those due to $\alpha_{n}^{2}=0$ and $\Delta_{n}=0$. However, the residue of the first term at $\alpha_{n}=0$ cancels with that due to the second term and hence the only significant poles are those of $\Delta_{n}=0$. Similar remarks apply in evaluating the residue of $U_{2}(r, x, s) \exp (s t)$. One obtains therefore

$$
\begin{aligned}
u_{1}(r, x, t)=\sum_{n} \frac{4 \pi \epsilon_{2}}{l^{2}} \frac{k_{1}-k_{2}}{k_{1} k_{2}} n \sin \frac{n \pi x}{l} \\
\cdot \sum_{j} \frac{\left\{I_{1}\left(\beta_{n j} a\right) K_{1}\left(\beta_{n j} b\right)-I_{1}\left(\beta_{n i} b\right) K_{1}\left(\beta_{n j} a\right)\right\} I_{0}\left(\alpha_{n j} r\right)}{\alpha_{n j}^{2} \beta_{n j} \Delta_{n}^{\prime}\left(\lambda_{n j}\right)} e^{\lambda_{n j} \iota},
\end{aligned}
$$

$$
\begin{aligned}
u_{2}(r, x, t)=\sum_{n} \frac{4 \pi \epsilon_{1}}{l^{2}} \frac{k_{1}-k_{2}}{k_{1} k_{2}} n \sin \frac{n \pi x}{l} \\
\cdot \sum_{j} \frac{\left\{I_{0}\left(\beta_{n i} r\right) K_{1}\left(\beta_{n j} b\right)-I_{1}\left(\beta_{n j} b\right) K_{0}\left(\beta_{n i} r\right)\right\} I_{1}\left(\alpha_{n j} a\right)}{\alpha_{n j} \beta_{n j}^{2} \Delta_{n}^{\prime}\left(\lambda_{n j}\right)} e^{\lambda_{n j} t},
\end{aligned}
$$

where $\lambda_{n i}$ are the zeroes of $\Delta_{n}(s)=0,(n=1,3,5, \cdots)$ and $\alpha_{n i}$ and $\beta_{n i}$ are the corresponding values defined by the relations (16) and (17) when $s$ has the values $\lambda_{n i}$.

The zeroes of $\Delta_{n}$ may be obtained from equation (20) by solving the equation

$$
\frac{\epsilon_{1} a_{n}}{\epsilon_{2} \beta_{n}} \frac{I_{1}\left(\alpha_{n} a\right)}{I_{0}\left(\beta_{n} a\right)}=\frac{I_{1}\left(\beta_{n} a\right) K_{1}\left(\beta_{n} b\right)-I_{1}\left(\beta_{n} b\right) K_{1}\left(\beta_{n} a\right)}{I_{0}\left(\beta_{n} a\right) K_{1}\left(\beta_{n} b\right)+I_{1}\left(\beta_{n} b\right) K_{0}\left(\beta_{n} a\right)}
$$

graphically. The equation (25) may be transformed into a form more suitable for numerical work by the substitutions

$$
\alpha_{n} a=i x, \quad \beta_{n} a=i y, \quad \beta_{n} b=i \rho y, \quad(\rho=b / a),
$$

$x$ and $y$ being related on account of (16) and (17) by the equation

$$
y^{2}=\sigma^{2}\left(x^{2}+n^{2} \pi^{2} a^{2} \delta^{2} / l^{2}\right)
$$


where $\sigma^{2}=k_{1} / k_{2}$ and $\delta^{2}=\left(k_{1}-k_{2}\right) / k_{1}$ are dimensionless constants. Introducing another dimensionless constant ${\sigma^{\prime 2}}^{2}=\epsilon_{1} / \epsilon_{2}$ and transforming the $I$ and $K$ functions into the corresponding $J$ and $Y$ functions* by the relations

$$
\begin{aligned}
I_{\nu}(i z) & =i^{\nu} J_{\nu}(z), \\
K_{\nu}(i z) & =i^{-\nu+1}\left[-J_{\nu}(z)+i Y_{\nu}(z)\right] \pi / 2,
\end{aligned}
$$

we obtain in place of (25)

$$
{\sigma^{\prime 2}}^{2} \frac{J_{1}(x)}{J_{0}(x)}=y \frac{J_{1}(\rho y) Y_{1}(y)-J_{1}(y) Y_{1}(\rho y)}{J_{1}(\rho y) Y_{0}(y)-J_{0}(y) Y_{1}(\rho y)} .
$$

Equation (27) has real roots and may be solved by plotting the right and left hand sides as functions of $x$. (Note that $y$ on the right hand side is not the corresponding ordinate, but determined by (26)).

Let

$$
x=\xi_{n i} \quad j=1,2,3, \cdots ; \quad n=1,3,5, \cdots
$$

be the roots of equation (27), the double subscript indicating that $\xi_{n i}$ is the $j$ th root of $\Delta_{n}=0$. Let the corresponding values of $\alpha_{n j} a$, etc. be

$$
\alpha_{n i} a=i \xi_{n i}, \quad \beta_{n i} a=i \sigma \eta_{n i}, \quad \beta_{n j} b=i \rho \sigma \eta_{n i},
$$

where

$$
\begin{gathered}
\eta_{n i}^{2}=\xi_{n i}^{2}+(n \pi a \delta / l)^{2} \text { by equation (26). Then } \\
\lambda_{n i}=-\left(\xi_{n i}^{2} / a^{2}+n^{2} \pi^{2} / l^{2}\right) k_{1}
\end{gathered}
$$

$u_{1}(r, x, t)$ and $u_{2}(r, x, t)$ can be now expressed in terms of $\xi_{n i}$ and $\eta_{n i}$ as follows:

$$
\begin{aligned}
& u_{1}(r, x, t)=\frac{8 \pi a^{3} \epsilon_{2}}{l^{2}} \frac{k_{1}-k_{2}}{k_{1} \sigma} \sum_{n} n \sin \frac{n \pi x}{l} \sum_{j} \frac{F_{11}\left(\rho \sigma \eta_{n i}, \sigma \eta_{n j}\right) J_{0}\left(\xi_{n j} r / a\right)}{\xi_{n j}^{2} \eta_{n j} D_{n}\left(\lambda_{n i}\right)} e^{\lambda_{n i} t}, \\
& u_{2}(r, x, t)=\frac{8 \pi a^{3} \epsilon_{1}}{l^{2}} \frac{k_{1}-k_{2}}{k_{1} \sigma^{2}} \sum_{n} n \sin \frac{n \pi x}{l} \sum_{j} \frac{F_{10}\left(\rho \sigma \eta_{n j}, \sigma \eta_{n i} r / a\right) J_{1}\left(\xi_{n j}\right)}{\xi_{n j} \eta_{n i}^{2} D_{n}\left(\lambda_{n i}\right)} e^{\lambda_{n i} t},
\end{aligned}
$$

where

$$
\begin{aligned}
D_{n}\left(\lambda_{n i}\right)=\frac{\epsilon_{2} a}{\sigma}\left(\frac{\eta_{n i}}{\xi_{n i}}-\sigma^{\prime 2} \frac{\xi_{n i}}{\eta_{n i}}\right) J_{1}\left(\xi_{n i}\right) F_{11}\left(\rho \sigma \eta_{n i}, \sigma \eta_{n j}\right) & \\
& +\frac{\epsilon_{1} b}{\sigma} \frac{\xi_{n i}}{\eta_{n i}} J_{1}\left(\xi_{n j}\right) F_{00}\left(\rho \sigma \eta_{n i}, \sigma \eta_{n j}\right) \\
& +\epsilon_{2} b J_{0}\left(\xi_{n j}\right) F_{10}\left(\sigma \eta_{n i}, \rho \sigma \eta_{n j}\right) \\
& +\frac{\epsilon_{1} a}{\sigma^{\prime 2}}\left(1-\frac{\sigma^{\prime 2}}{\sigma^{2}}\right) J_{0}\left(\xi_{n i}\right) F_{01}\left(\sigma \eta_{n j}, \rho \sigma \eta_{n i}\right), \\
& F_{\mu \nu}=J_{\mu}(x) Y_{\nu}(y)-J_{\nu}(y) Y_{\mu}(x) .
\end{aligned}
$$

${ }^{*}$ Ref. [3], Appendix III. 
Verification of the solution.* As the series solution established by the Laplace transform method is purely formal, it is necessary to show that it satisfies all the conditions of the boundary value problem and is unique. It is obvious that the series solutions (28) and (29) for $u_{1}(r, x, t)$ and $u_{2}(r, x, t)$ respectively satisfy the boundary conditions (4) and (5). It is also seen that the boundary conditions (7) and (8) are satisfied by direct substitution of the expressions for $u_{1}$ and $u_{2}$, and (6) is satisfied on account of the relation $\Delta_{n}\left(\lambda_{n i}\right)=0$. It only remains, therefore, to verify that the initial condition, viz., $u_{1}=u_{2}=1$ for $t=0$. This is done most conveniently with the contour integral form of the solutions (21) and (22). Consider $u_{1}$ for example.

For $t=0$ we have from (18) and (21)

$$
u_{1}(r, x, 0)=\sum_{n} \frac{4}{n \pi} \sin \frac{n \pi x}{l}+\sum_{n} \frac{4}{n \pi} \sin \frac{n \pi x}{l} \cdot \frac{1}{2 \pi i} \int_{\gamma-i \infty}^{\gamma+i \infty} A_{n} I_{0}\left(\alpha_{n} r\right) d s
$$

where

$$
A_{n}=\frac{\epsilon_{2}\left(k_{1}-k_{2}\right)}{k_{1} k_{2}}\left(\frac{n \pi}{l}\right)^{2} \frac{I_{1}\left(\beta_{n} a\right) K_{1}\left(\beta_{n} b\right)-I_{1}\left(\beta_{n} b\right) K_{1}\left(\beta_{n} a\right)}{\beta_{n} \alpha_{n}^{2} \Delta_{n}(s)} .
$$

since

$$
\sum_{n} \frac{4}{n \pi} \sin \frac{n \pi x}{l}=1 \quad \text { we may write } \quad u_{1}=1+v_{1}
$$

where

$$
v_{1}=\sum_{n} \frac{4}{n \pi} \sin \frac{n \pi x}{l} \frac{1}{2 \pi i} \int_{\gamma-i \infty}^{\gamma+i \infty} A_{n} I_{0}\left(\alpha_{n} r\right) d s .
$$

It thus suffices to show that $v_{1} \equiv 0$. The path of integration is a straight line parallel to the imaginary axis such that all the poles of the integrand lie to the left of this line. As the poles $\lambda_{n i}$ are all negative we can choose the path with any $\gamma>0$. We shall choose $\gamma$ large and positive. Since $\alpha_{n}^{2}=s / k_{1}+n^{2} \pi^{2} / l^{2}$ and $\beta_{n}^{2}=s / k_{2}+n^{2} \pi^{2} / l^{2}$ it is clear that $\left|\alpha_{n}^{2}\right|$ and $\left|\beta_{n}^{2}\right|$ will be large both for large $\xi$ and large $\eta$. Further, if $k_{1}>k_{2}$ (say) we have on the path of integration $\left|\alpha_{n}\right|<\left|\beta_{n}\right|$.

Replacing now the modified Bessel functions in equation (33) for $A_{n}$ by their asymptotic expansions for large argument and retaining only the dominant terms in the numerator and denominator we find that the integral in (34) becomes, apart from a constant factor

$$
n^{2}\left(\frac{a}{r}\right)^{1 / 2} \int_{\gamma-i \infty}^{\gamma+i \infty} \frac{e^{-\alpha_{n}(a-r)}}{\epsilon_{2} \beta_{n}+\epsilon_{1} \alpha_{n}} \frac{d s}{\left(\alpha_{n} \beta_{n}\right)^{3 / 2}} .
$$

It may be shown that the absolute value of this expression is less than

$$
2\left(\epsilon_{1}+\epsilon_{2}\right)\left(\frac{a}{r}\right)^{1 / 2} n^{2} \exp \left\{-\left(\frac{\gamma}{2 k_{1}}+\frac{n^{2} \pi^{2}}{2 l^{2}}\right)(a-r)\right\} \int_{0}^{\infty} \frac{d \eta}{\left(P^{2}+Q^{2} \eta^{2}\right)^{1 / 2}\left|\alpha_{n}\right|^{3 / 2}\left|\beta_{n}\right|^{1 / 2}}
$$

Thus the absolute values of the terms of the series in (34) are majorized by

$$
c_{1}\left(\frac{a}{r}\right)^{1 / 2} \exp \left\{-\frac{r}{2 k_{1}}(a-r)\right\} \sum_{n} K(n) \exp \left\{-\frac{n^{2} \pi^{2}}{2 l^{2}}(a-r)\right\}
$$

*Ref. [3], Appendix I. 
where $K(n)$ is $0(n u)$ with a fixed finite $\mu$. The expression (35) shows that $v_{1}$ can be made arbitrarily small by making $\gamma$ large. Hence it follows that $v_{1} \equiv 0$. Similar reasoning shows that $u_{2}$ also satisfies the initial condition.

The proof of the uniqueness of the solution is well known and need not be repeated here.

A numerical example. To illustrate the numerical procedure, the temperature distribution in a composite cylinder having the following parameters is calculated

$$
\begin{aligned}
& k_{1}=1, \quad k_{2}=0.1, \quad \epsilon_{1}=0.4, \quad \epsilon_{2}=0.04 \\
& a=1, \quad b=1.5, \quad l=10 ; \quad u_{1}(r, x, 0)=u_{2}(r, x, 0)=1
\end{aligned}
$$

Equation (27) leading to the roots $\xi_{n i}$ in this particular case is plotted in Fig. 2.

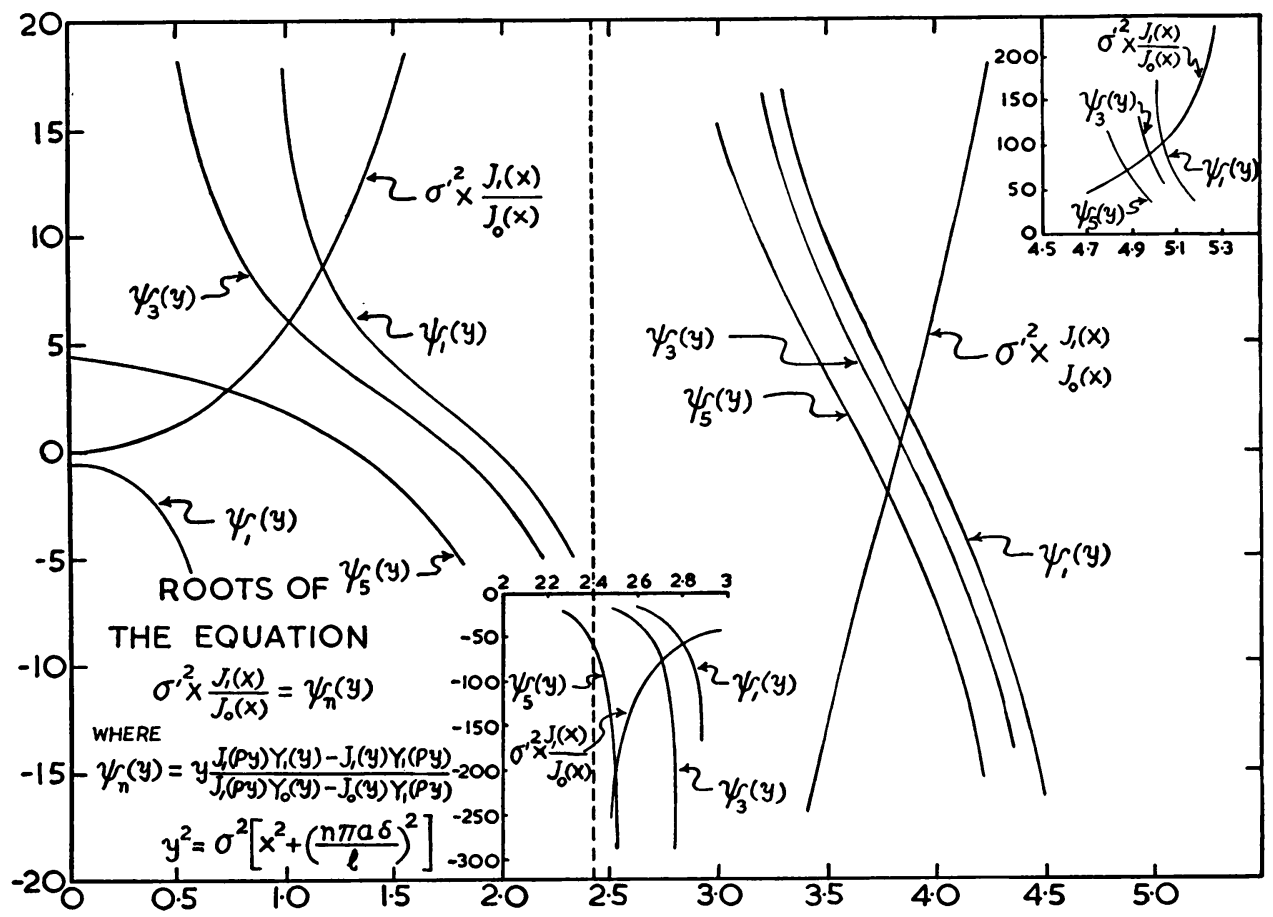

FIG. 2. Graphical solution of the first few roots of the eq. 27 for $n=1,3,5$.

\begin{tabular}{|c|c|c|c|c|}
\hline & 1 & 2 & 3 & 4 \\
\hline 1 & 1.18 & 2.83 & 3.88 & 5.04 \\
\hline 3 & 1.03 & 2.74 & 3.85 & 4.98 \\
\hline 5 & 0.75 & 2.52 & 3.78 & 4.88 \\
\hline
\end{tabular}

The first few roots are given in the table below. 


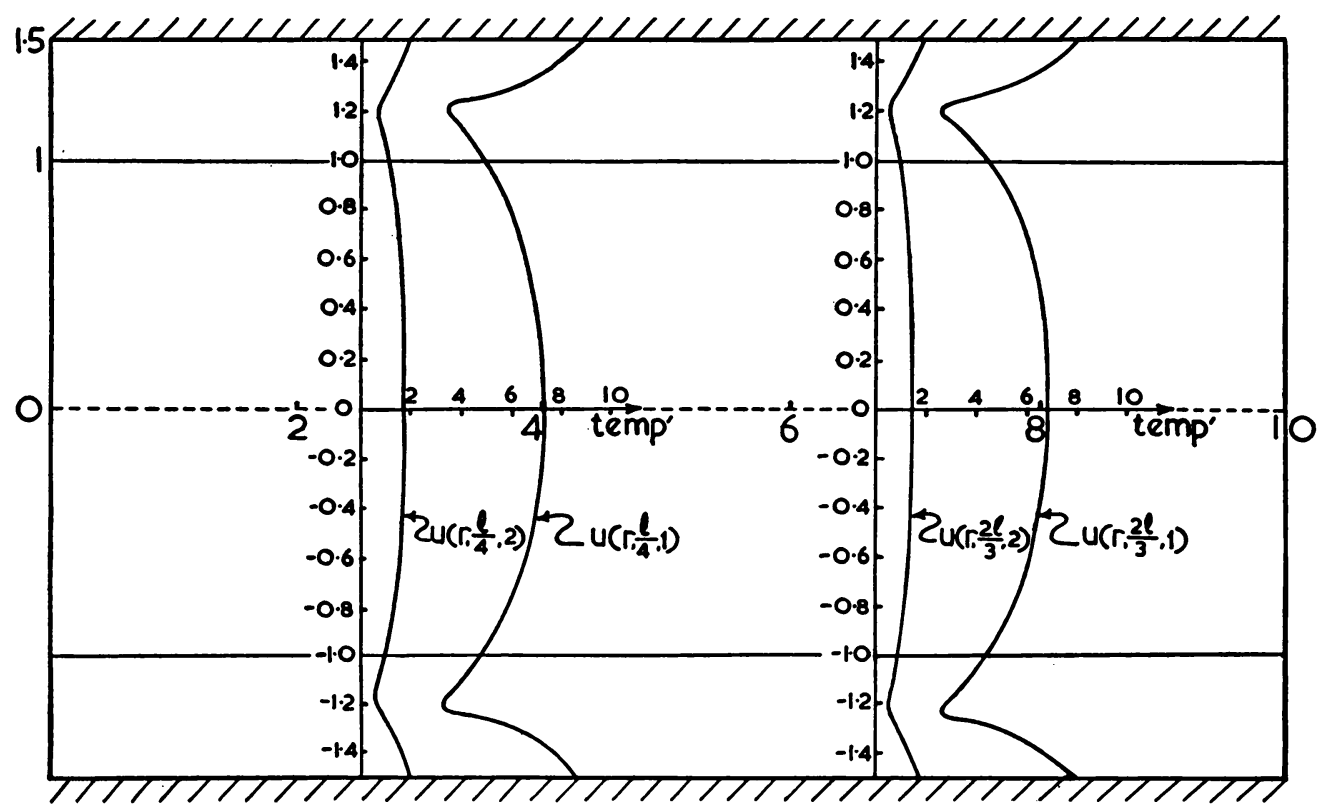

FIG. 3. Distribution of temperature in the composite cylinder along the radius at $x=l / 4$ and $x=2 l / 3$ for $t=1$ and $t=2$ secs.

The distribution of temperature along the radius at $x=2 l / 3$ and $l / 4$ for $t=1$ sec. and 2 secs. is plotted in Fig. 3.

\section{BiBLIOGRAPHY}

1. J. C. Jaeger, Heat conduction in composite circular cylinders, Phil. Mag. (213) 32, 324-335 (1941).

2. L. P. Smith, Heat flow in an infinite solid bounded internally by a cylinder, Jour. App. Phys. (6) 8, 441-448 (1937).

3. H. S. Carslaw and J. C. Jaeger, Conduction of heat in solids, Oxford, 1947, Art. 119.

4. W. J. Jacobi, Propagation of sound waves along liquid cylinders, Jour. Acous. Soc. Amer. (2) 21, 120-127 (1949).

5. L. Pincherle, Electromagnetic waves in metal tubes filled longitudinally with two dielectrics, Phys. Rev. $(5,6) 66,118-130$ (1944).

6. R. D. Teasdale and T. J. Higgins, Electromagnetic waves in circular wave guides containing two coaxial media, Proc. National Electronics Conference (USA), 5, 427-441 (1949).

7. R. V. Churchill, Modern operational methods in engineering, McGraw Hill, 1944, p. 157. 